\section{Evaluación de la eficacia y seguridad de la inyección intravítrea de acetónido de triamcinolona en el tratamiento del edema de mácula difuso del diabético Efficacy and safety of intravitreal injection of triamcinolone acetonide as treatment for diffuse diabetic macular edema}

En 2005 se inició uno de los primeros ensayos clínicos independientes de la Oftalmología española (1).

En aquel momento y aun hoy, se utilizan las inyecciones intraoculares de acetónido de triamcinolona sin una base científica suficientemente probada orientados por casos clínicos aislados o series demasiado cortas por lo que la Red Temática 03/13 dirigida por el Profesor Sánchez Salorio propuso al Instituto Carlos III la realización de este ensayo. A él se sumaron centros de Madrid (Complutense y Clínico de San Carlos), Barcelona (Vall de Hebron), Alicante (Instituto Oftalmológico), Murcia (Universidad y Hospital), Santiago de Compostela (INGO y Hospital Universitario), Clínica Universitaria de Navarra y Valladolid (IOBA y Hospital Clínico) coordinados por el IOBA que ha actuado como promotor del ensayo.

La idea fue comparar la eficacia del láser en rejilla frente a un grupo de pacientes al que un mes después de la inyección de $4 \mathrm{mg}$ de triamcinolona, y aprovechando la reducción que se produce en el espesor retiniano se aplicó el mismo tratamiento. Se solicitó el numero EUDRA (CT:2005-001385-14) y el proyecto se inscribió en la base de ensayos del National Institute of Health (NCT:00309192).

El 23 de enero de 2006 tras la aprobación de la Agencia Española del Medicamento se comenzó este ensayo prospectivo, randomizado y en paralelo.

Y al inicio de 2009 se ha concluido el trabajo, que ha tenido numerosos problemas que han sido pacientemente solventados gracias al entusiasmo de los investigadores. Se han incluido el $60 \%$ de los pacientes programados (57 de 98 ) y éstas son sus principales conclusiones:

- Se observa una mayor reducción del espesor retiniano en el grupo de triamcinolona.

- No hay diferencias significativas en cuanto a la agudeza visual.

- A los 6 meses hay un 30\% más de cataratas en el grupo con triamcinolona.
- En este grupo 4 pacientes sufrieron aumentos de la presión intraocular y uno requirió tratamiento aunque todos fueron sometidos al test de provocación con dexametasona antes de ser incluidos.

- No ha habido ninguna endoftalmitis ni efectos adversos graves

A la vista de este trabajo es cuestionable seguir utilizando este tratamiento para este tipo de pacientes (edemas quísticos y malas visiones) por lo que desde la nueva Red Temática, no se recomienda su utilización.

En este intervalo de tiempo han aparecido otros trabajos de los que destacamos el realizado por la Diabetic Retinopathy Clinical Research Network (2) con 840 ojos. Sus resultados indican que a los dos años el láser es más efectivo y tiene menos efectos adversos que las inyecciones de $1 \mathrm{mg}$ o 4 $\mathrm{mg}$ de triamcinolona, sin preservantes.

Y aunque hay algunos trabajos que hablan a favor de su uso (3) la contundencia del realizado por el DRC.net deja muy claro que la triamcinolona intravítrea solo produce una mejoría pasajera en el edema macular diabético y que a largo plazo no ofrece mejores resultados funcionales que el láser, con el agravante de que aumenta de forma significativa las complicaciones.

Además de las conclusiones anteriores, hay otras experiencias muy positivas de este trabajo multicéntrico que han supuesto un impulso a otros ensayos que están ahora en marcha.Y sobre todo la Oftalmología española ha demostrado que puede trabajar en red y concluir los trabajos, superando para ello todas las dificultades.

López-Gálvez MI, Pastor-Jimeno JC

Doctor en Medicina

Instituto Universitario de Oftalmobiología Aplicada de la Universidad de Valladolid.

Valladolid. España

pastor@ioba.med.uva.es

\section{BIBLIOGRAFÍA}

1. Pastor JC. Investigación clínica independiente: es posible. Arch Soc Esp Oftalmol 2006; 81: 133-134.

2. Beck RW, Edwards AR, Aiello LP, Bressler NM, Ferris F, Glassman AR, et al. Triamcinolone acetonide and Focal/Grid photocoagulation for diabetic macular edema. Ophthalmology 2008; 115: 1447-1459.

3. Maia O, Takahashi B, Costa R, Scott I, Takahashi W. Combined laser and intravitreal triamcinolone for proliferative diabetic retinopathy and macular edema: one-year results of a randomized clinical trial. Am J Ophthalmol 2009; 147: 291-297. 